\title{
Uncommon Dislocation Processes at the Incipient Plasticity of Stepped Gold Surfaces
}

\author{
V. Navarro, O. Rodríguez de la Fuente, A. Mascaraque, and J. M. Rojo \\ Dto. de Física de Materiales, Universidad Complutense de Madrid, 28040 Madrid, Spain
}

(Received 30 August 2007; published 14 March 2008)

\begin{abstract}
Gold vicinal surfaces (788), with a high density of steps, along with (111) flat surfaces taken as a reference, have been nanoindented and their resulting penetration curves and related defect structure comparatively analyzed by AFM and atomistic simulations. Stepped surfaces are shown to yield at smaller loads than (111) ones in agreement with calculations of the critical resolved shear stress needed to nucleate a dislocation. In the stepped surfaces, a novel intermediate state is identified in which the penetration curves depart from a Hertzian behavior prior to the appearance of pop-ins. This state is shown to result from heterogeneous nucleation at preexisting surface steps of dislocation loops, most of which retract and vanish when the indenter load is removed.
\end{abstract}

DOI: 10.1103/PhysRevLett.100.105504

PACS numbers: 62.20.F-, 68.37.Ef, 79.60.-i

Nanoindentation, in conjunction with real-space techniques such as Atomic Force Microscopy (AFM) or Transmission Electron Microscopy (TEM), provides basic information about surface mechanical properties and, in particular, about the elastic-plastic transition at the nanoscale. Experiments and simulations [1-8] have resulted in a general comprehension of surface plasticity. However, in spite of much effort devoted to their understanding, the incipient stages of plasticity have proved complex, and a number of important questions still need to be elucidated.

The present Letter addresses two controversial issues in this field. The first one regards the processes related to the nucleation of dislocations in the earliest stages of surface deformation. Recent experimental [9] and simulation [10] works suggest that, contrary to the classical picture generally accepted, some kind of dislocation activity actually precedes the appearance of discontinuities (baptized popins) in the force $(F)$ vs penetration depth $(\delta)$ curve. The second issue regards the role of preexisting surface defects, often ignored when analyzing surface mechanical properties. Real surfaces are far from ideal: they are rich in different kinds of surface defects, which can play a substantial role in the surface response to mechanical probes. Understanding their role can also unravel a number of problems in the fields of contact mechanics and friction between rough solids. Some preliminary studies [2-4,1113] have found that steps on metal surfaces play an active role by decreasing the elastic threshold and assisting the nucleation of dislocations. On the other hand, collective effects based on interactions involving preexisting defects can lead to hardening phenomena [14]. All these issues have profound implications in plasticity models [15].

Here, we have worked with vicinal surfaces, which provide an average over a number of steps and can be seen as model defective surfaces. In this Letter, we present results which combine experimental observations and atomistic simulations of the mechanical properties of stepped $\mathrm{Au}(788)$ vicinal surfaces using the corresponding flat $\mathrm{Au}(111)$ surface as a reference. Atomistic simulations of the nanoindentation processes are carried out providing both $F$ vs $\delta$ curves and maps of atomic displacements around the nanoindentation point. The different experimental yield strengths observed in the vicinal and flat surfaces are analyzed in terms of atomic mechanisms involving preexisting surface defects. Near the threshold of plasticity, a novel mechanism is disclosed, in which dislocation loops are heterogeneously nucleated at the steps. These loops apparently do not give rise to pop-ins in the penetration curves; moreover, they draw back and vanish once the tip is retracted without leaving any permanent traces in the AFM images. This is an illustrative example of heterogeneously nucleated dislocation activity preceding pop-in generation.

Two atomic force microscopes (from Veeco and Nanotec) were used both to perform nanoindentations and to take images of the surfaces, the data being analyzed with the WSXM software package [16]. A diamond tip glued to a steel cantilever was used to get the cantilever deflection curves because of its high hardness and low compliance. A diamond single crystal was used as a reference to get the real penetration in the $F$ vs $\delta$ curves. The gold samples were oriented with a misalignment smaller than $0.1^{\circ}$ from the ideal (788) direction, ion bombarded and annealed at high temperature in ultra high vacuum before the experiments performed under ambient conditions. The splitted spots of the LEED pattern confirmed the regular separation of the steps. Atomistic simulations with approximately $1.5 \times 10^{6}$ atoms were carried out using the embedded atom potential [17]. The indenter was modeled as a spherical purely repulsive potential. It was approached towards the surface in small increments and the potential energy of the whole system minimized in every step with a conjugate gradient algorithm [18]. Lateral $x y$ periodic boundary conditions were applied, while the bottom layer was kept fixed in the $z$ direction. The whole simulation cell (with lateral sizes of 39 and $37 \mathrm{~nm}$ ) was rescaled in order to keep the diagonal elements of the stress tensor $\sigma_{x x}$ and $\sigma_{y y}$ equal to zero. The ATOMEYE software [19] was used as a 
visualizing tool, which allowed us to monitor subsurface defect evolution during the simulation. A similar combination of experiment and computation helped us in the past $[5,20,21]$ to obtain unambiguous identification of dislocation configurations produced by nanoindentation experiments.

The Au(788) surface ideally consists of (111) terraces $3.9 \mathrm{~nm}$ wide separated by monoatomic steps of the $\{111\}$ type towards the [ $\overline{2} 11]$ azimuthal direction. This surface is misoriented by $3.5^{\circ}$ with respect to the (111) orientation. Parallel steps run along the [0 $\overline{1} 1]$ direction [22]. In Fig. 1(a), we depict a scheme of the stepped surface together with the AFM tip, showing that our experiment effectively probes several steps. Figure 1(b) shows an image of a $\mathrm{Au}(788)$ surface after being indented with a $3 \times$ 3 indentation matrix, the same load having been applied for all the indentations. Note the excellent reproducibility of the traces, both in size and shape. When nanoindentations are performed with increasing load, the size of the permanent traces increases with the external force (not shown). In all cases (flat and vicinal surfaces), below a certain threshold corresponding to the appearance of pop-ins in the penetration curves, there are no visible permanent traces in the AFM images after the nanoindentation.

In Fig. 2, we have represented typical examples of $F$ vs $\delta$ curves for the flat $\mathrm{Au}(111)$ and stepped $\mathrm{Au}(788)$ surfaces. In the flat one, two different stages are recognized: an initial reversible stage, for low penetrations, in which the curves can be fitted to a Hertzian behavior [23] and a second stage, when the surface deformation appears irreversible and where the retraction of the curve shows a hysteretical behavior, as shown in the figure inset. In this second stage, the $F$ vs $\delta$ curve immediately exhibits popins. The transition point between the two stages, is identified as the yield point (YP). For the stepped (788) surface, the $F$ vs $\delta$ curve shows a qualitatively different behavior: the deviation from a Hertzian response, starting at point A,
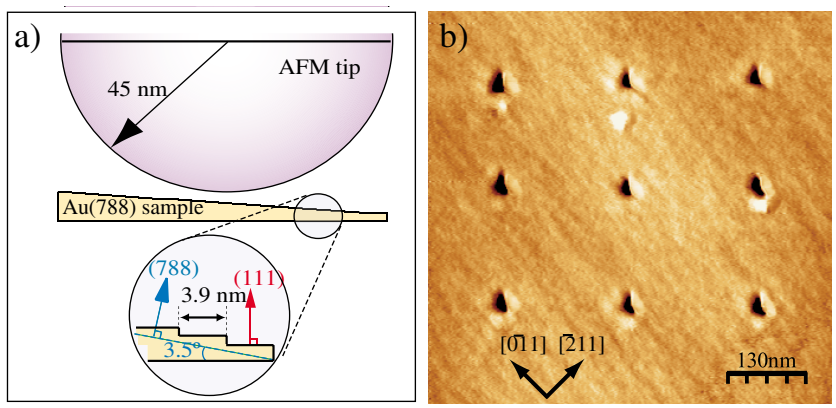

FIG. 1 (color online). (a) Schematic and scaled model of the tip-sample system showing the relationship between the tip and steps sizes. (b) AFM image of $3 \times 3$ nanoindentation matrix on the $\operatorname{Au}(788)$ surface. The nine traces left on the surface are clearly visible, as well as the piled-up material around them. Bands associated with the steps are apparent along the [011] direction. is continuous, and pop-ins only appear in a third later stage, from point $B$ onwards.

The initial reversible stage of either curve in Fig. 2 corresponds to the elastic regime and can be fitted to a Hertzian behavior $F=(4 / 3) R^{1 / 2} E^{*} \delta^{3 / 2}$ where $R$ is the tip radius $(R=45 \mathrm{~nm})$ and $E^{*}$ is the reduced Young modulus, which depends on the Young modulus $E$ and Poisson ratio of sample and tip [23]. The fitted values of $E^{*}$ are clearly higher for the flat surface $E^{*}(111)=61 \pm 3 \mathrm{GPa}$ than for the stepped one $E^{*}(788)=50 \pm 2 \mathrm{GPa}$, the main source of error being the location of the zero force. This does not necessarily imply that the intrinsic value of $E$ is lower for the stepped surface. Other factors may contribute: for example, at the early stages of contact, surface defects tend to reduce the effective contact area.

Two significant results related to the onset of plasticity can be deduced from Fig. 2. The first one is that stepped surfaces yield earlier, in the sense that they depart from a Hertzian elastic behavior under a lower load than flat surfaces do. Taking their respective yield points for each surface as YP (flat) and A (stepped), $F_{\text {yield }}(111)=750 \pm$ $240 \mathrm{nN}$ and $F_{\text {yield }}(788)=465 \pm 130 \mathrm{nN}$. In terms of the Hertz model, the maximum resolved shear stress (in a continuum) is $\tau=0.31 p_{0}$, where $p_{0}=\left[\left(6 F E^{* 2}\right) /\left(\pi^{3} R^{2}\right)\right]^{1 / 3}$ is the maximum normal stress [23]. Using the above yield values, we immediately obtain $\tau(111)=2.1 \pm 0.2 \mathrm{GPa}$ and $\tau(788)=1.6 \pm 0.2 \mathrm{GPa}$. Note that the value for the flat surface agrees with earlier measurements $[2,3,20]$ and is of the order of the theoretical shear stress for an ideal crystal without defects. Panel (b) of Fig. 2 shows a distribution of the yield points, YP and $A$, for many different experiments both on the flat and the stepped surfaces. Nanoindentation properties at the nanometer scale are ex-
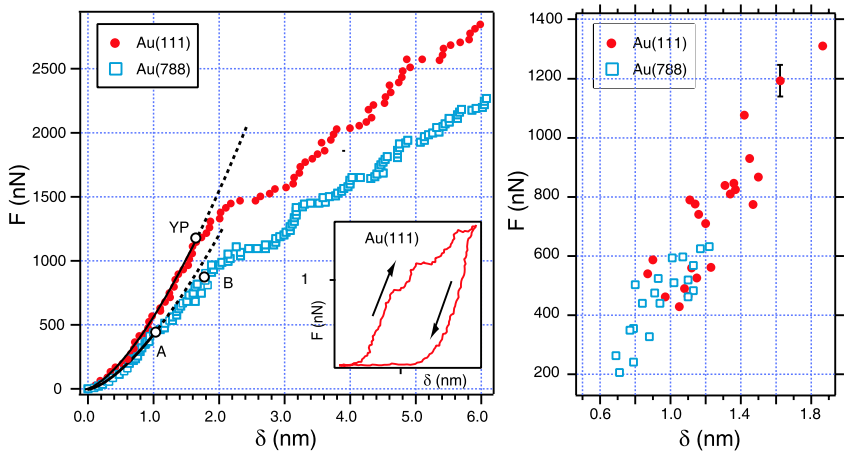

FIG. 2 (color online). (a) Comparison of $F$ vs $\delta$ curves for a flat (closed circles) and a stepped (open squares) surface. Both surfaces show a first elastic stage (fitted to a Hertzian $F \sim \delta^{3 / 2}$ behavior, dotted lines), which terminates at point YP in the flat surface and at point $A$ in the stepped surface (see text for details). Inset: detail of another $\mathrm{Au}(111)$ curve showing the return branch of the curve. (b) Distribution of the termination points of the elastic stage for both surfaces: point YP for the (111) surface (closed circles) and point $A$ for the (788) orientation (open squares). 
pected to show a large variation from test to test, due not only to surface inhomogeneities but to the intrinsically statistical nature of the underlying processes [8]. The scattering of the values is large, but smaller for the stepped $(130 \mathrm{nN})$ than for the flat surface $(240 \mathrm{nN})$ because the existence of previous surface defects is more critical on the flat surface than on the stepped one [24].

The second significant result arises from a comparison of the shapes of the curves for the flat and stepped surface beyond their yield points in Fig. 2. In the stepped surface, the deviation from the elastic Hertzian regime takes place following a smooth curve without any visible pop-in. Popins only emerge beyond a certain point $B$ for forces, of the order of $1000 \mathrm{nN}$. In that intermediate stage between points $A$ and $B$ of Fig. 2(a), AFM images do not show any permanent traces after tip retraction. We identify this novel intermediate stage as a nonelastic stage without discontinuous pop-ins, which is reversible in the sense that there are no permanent traces left.

The nature of this uncommon intermediate stage is clarified by the simulations. The simulated nanoindentation on the flat surface (lower panel of Fig. 3) shows the expected behavior [20]: the elastic range fits quite well to a Hertzian shape, up to a point where a critical value of the resolved shear stress $\sigma_{r}$ is reached and an abrupt jump is accompanied by the creation of multiple dislocation loops in all the four $\{111\}$ slip planes of the fcc crystal [25]. This point corresponds to the yield point YP of Fig. 2. However, the simulated stepped (788) surface shows quite a different behavior. The penetration curve always runs below that of the flat surface and deviates from a Hertzian behavior about halfway before point $B$. Although an abrupt pop-in excursion appears at $\delta \approx 11.6 \AA$, much dislocation activity is present before it. When defects are tracked in real space, dislocation loops are seen to nucleate at the steps as soon as the indenter comes into contact with a step, without any associated pop-in excursion. All the atoms in the step are pushed in a continuous way, nucleating a partial dislocation and its corresponding stacking fault in the slip direction. Only the two slip planes parallel to the step line are active: the $(1 \overline{1} \overline{1})$ and the (111) planes. All these dislocation processes take place without any associated popin excursion and are observed to be reversible [25], i.e., once the tip is retracted, the resulting dislocation damage rearranges and disappears [26]. Furthermore, there is no trace left, in agreement with experimental AFM results referred above. We identify this behavior with the experimental intermediate range. Because only some of the characteristics of ordinary plasticity are met, we design this range as quasiplastic. The transition from quasiplasticity to normal plasticity, which corresponds to the experimental point $B$, is marked by the appearance of a pop-in in the simulated $F$ vs $\delta$ curve and by the emergence of many new dislocations, some of these being the result of the cross slip, multiplication, and movement of earlier dislocations.
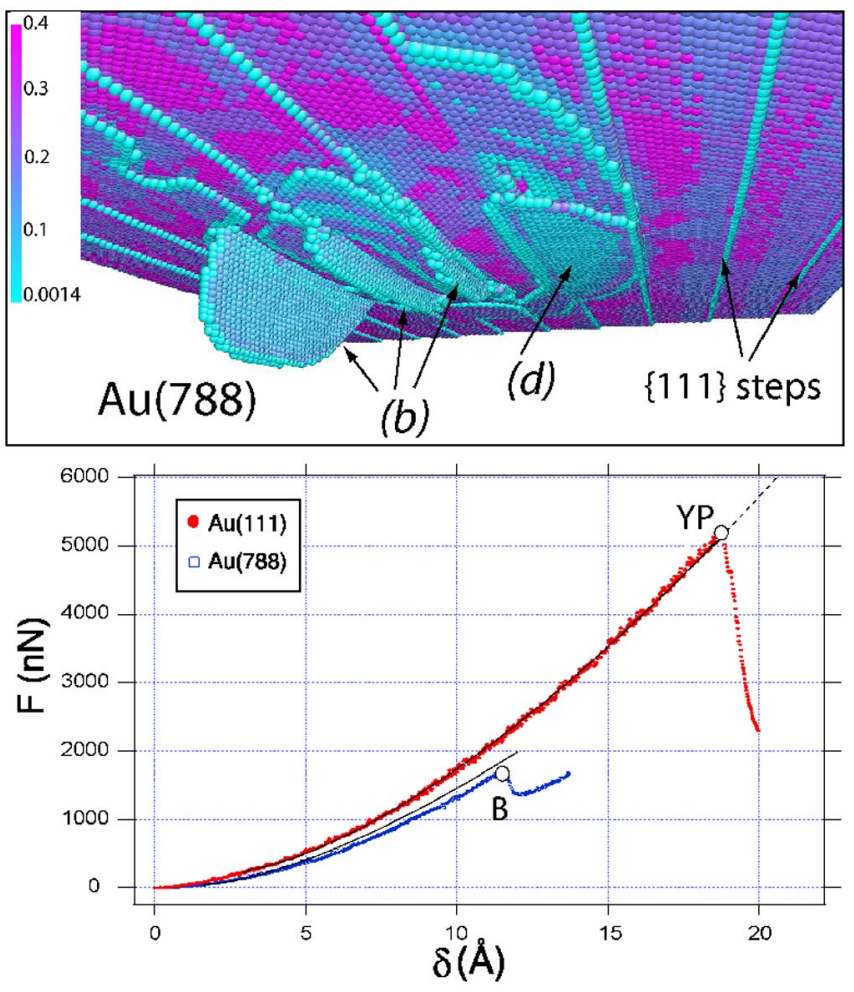

FIG. 3 (color online). Top: subsurface view of the generated defect structure in the stepped $\mathrm{Au}(788)$ surface during the intermediate stage. Only surface and defective atoms are displayed, according to their centrosymmetric parameter (see bar scale) [19]. Light intensity designs stacking atom faults and partial dislocations. This view corresponds to a penetration $\delta=$ $11.4 \AA$, as indicated by an arrow in the panel below. Two kinds of partial dislocations half-loops (together with their corresponding staking faults) extend along the two slip planes parallel to the steps: $(1-1-1)$ and (111) [(b) and (d) in Thompson notation, respectively]. Note that all defects at this plastic stage protrude from the step lines. Bottom: simulated indentation curves for a flat $\mathrm{Au}(111)$ (dashed line) and for stepped $\mathrm{Au}(788)$ (solid line) surfaces. In both cases, the fit to the Hertz model is represented as black lines. Note the deviation for the $\mathrm{Au}(788)$ while it perfectly fits for the flat $\mathrm{Au}(111)$ one.

Now, the defects do not necessarily originate at the steps, nor are they restricted to extend along the two slip planes parallel to the steps. They are also permanent, in the sense that most of them stay in the crystal once the tip is retracted.

Analysis of the simulation data also helps to understand the mechanisms underlying the observed lower yield strength of stepped surfaces. We can write the atomic resolved shear stress, $\sigma_{r}=\hat{b} \cdot \tilde{\sigma} \cdot \hat{n}$, in terms of the atomic stress tensor $\tilde{\sigma}$ where $\hat{b}$ and $\hat{n}$ are the unitary vectors of the slip vector and plane normal, respectively. We compute $\sigma_{r}$ for the atoms that nucleate a dislocation, just before the plane slips. For the homogeneous case below the flat surface, the first dislocation nucleates when $\sigma_{r} \approx 4 \mathrm{GPa}$. 
In the case of the stepped surface, the slip of the atoms below the step occurs before $\sigma_{r} \approx 2 \mathrm{GPa}$.

Although the general trends of the penetration curves are well explained by the simulations, there remain numerical discrepancies between simulated and measured values. They are probably connected with the rather crude assumptions underlying the present simulations, among others: (i) ignoring the role of temperature even if dislocation nucleation is a stress-biased thermally activated process [8] (in fact, with these simulations, we are exploring the upper limit of the yield strength), (ii) assuming an inert indenter (spherical, repulsive, and frictionless), (iii) assuming ideal surfaces, in the sense that either no defects are present at all (flat surface) or the step morphology and distribution is perfectly regular (stepped surface) [27].

Recent studies argue that, before any pop-in appears, substantial dislocation activity is detected $[9,10]$. Our results indicate that, during an intermediate stage, activity of this type is indeed present in stepped surfaces. In these surfaces, the elastic stage is ended by a continuous generation of defects, of which heterogeneous dislocation loops nucleating at the steps are predominant. The slip planes of these loops are determined by the step orientation, a fact which might regulate, during further deformation, the spatial distribution and mutual interactions between segregated dislocations leading to collective behaviors [14]. As simulation shows, these first defects are not stable, and consequently, although nonelastic, this section of the penetration curve is practically reversible. Although in ideal surfaces the first pop-in may be related to the nucleation of the first dislocation, our results show that this is not the case in real surfaces, where a certain plastic activity exists before any pop-ins. It appears to be catalyzed by heterogeneous dislocation nucleation at surface irregularities.

In conclusion, we have shown that the mechanisms involved in the incipient stages of plasticity induced by nanoindentation are different in flat and vicinal gold surfaces. Vicinal surfaces yield earlier than flat orientations due to preferential dislocation nucleation at the surface steps. Our calculations show that the critical stress for dislocation nucleation at the steps is approximately half that on the flat surface. Most of these dislocations are metastable under stress and evanesce when the indenting tip is retracted. Associated to this type of nucleation, we have revealed a novel intermediate stage in the $F$ vs $\delta$ curves characterized by a continuous deviation from an elastic behavior while being reversible and not resulting in pop-ins. Our work gives an example of dislocation nucleation and motion preceding pop-in excursions in the incipient plastic stages of a realistic surface, provides a clear example of heterogeneous defect nucleation under mechanical stress, and paves the way for a better understanding of contact mechanics in real surfaces.

The authors thank J.E. Ortega for providing us the $\mathrm{Au}(788)$ sample. Financial support from the Spanish MEC, Project No. MAT2006-13149-C02-01 and from the CAM Projects Nos. CAM-S-0505/PPQ/0316 and GR/ MAT/0632/2004 are gratefully acknowledged. V. N. thanks MEC through No. MAT2003-08627-C02-01.

[1] J. Li et al., Nature (London) 418, 307 (2002).

[2] S. G. Corcoran et al., Phys. Rev. B 55, R16057 (1997).

[3] J.D. Kiely and J.E. Houston, Phys. Rev. B 57, 12588 (1998); J. D. Kiely, R. Q. Hwang, and J. E. Houston, Phys. Rev. Lett. 81, 4424 (1998).

[4] A. Gouldstone, K. V. Vliet, and S. Suresh, Nature (London) 411, 656 (2001).

[5] O.R. de la Fuente et al., Phys. Rev. Lett. 88, 036101 (2002).

[6] C. L. Kelchner, S. J. Plimpton, and J. C. Hamilton, Phys. Rev. B 58, 11085 (1998).

[7] G. L. W. Cross et al., Nat. Mater. 5, 370 (2006).

[8] C. A. Schuh, J. K. Mason, and A. C. Lund, Nat. Mater. 4, 617 (2005).

[9] A. M. Minor et al., Nat. Mater. 5, 697 (2006).

[10] J. Knap and M. Ortiz, Phys. Rev. Lett. 90, 226102 (2003).

[11] J.A. Zimmerman et al., Phys. Rev. Lett. 87, 165507 (2001).

[12] D. Shan et al., Mater. Sci. Eng. A 412, 264 (2005).

[13] M. R. Shankar, Appl. Phys. Lett. 90, 171924 (2007).

[14] H. H. Yu et al., J. Mech. Phys. Solids 55, 489 (2007).

[15] J. Li, MRS Bull. 32, 151 (2007).

[16] I. Horcas et al., Rev. Sci. Instrum. 78, 013705 (2007).

[17] S. M. Foiles, Phys. Rev. B 32, 3409 (1985).

[18] W.H. Press et al., Numerical Recipes in Fortran (Cambridge University Press, Cambridge, 1992).

[19] J. Li, Model. Simul. Mater. Sci. Eng. 11, 173 (2003).

[20] A. Asenjo et al., Phys. Rev. B 73, 075431 (2006).

[21] E. Carrasco et al., Phys. Rev. B 68, 180102 (2003).

[22] A. Mugarza et al., Phys. Rev. Lett. 87, 107601 (2001).

[23] K. L. Johnson, Contact Mechanics (Cambridge University Press, Cambridge, 1985).

[24] Y. Shibutani and A. Koyama, J. Mater. Res. 19, 183 (2004).

[25] Movies of these processes are available as Auxiliary Material. See EPAPS Document No. E-PRLTAO-100024811. For more information on EPAPS, see http:// www.aip.org/pubservs/epaps.html.

[26] Except a few dislocations at the surface, with Burgers vector parallel to the surface.

[27] B. Luan and M. O. Robbins, Nature (London) 435, 929 (2005). 